\title{
In Silico Prediction and Insights Into the Structural Basis of Drug Induced Nephrotoxicity
}

\author{
Yinping Shi ${ }^{1}$, Yuqing Hua ${ }^{1,2}$, Baobao Wang $^{3}$, Ruiqiu Zhang ${ }^{1,2}$ and Xiao $\mathrm{Li}^{1,4 *}$ \\ ${ }^{1}$ Shandong Medicine and Health Key Laboratory of Clinical Pharmacy, Department of Clinical Pharmacy, The First Affiliated \\ Hospital of Shandong First Medical University and Shandong Provincial Qianfoshan Hospital, Jinan, China, ${ }^{2}$ School of Pharmacy, \\ Shandong First Medical University, Tai'an, China, ${ }^{3}$ Department of Nephrology, The First Affiliated Hospital of Shandong First \\ Medical University and Shandong Provincial Qianfoshan Hospital, Jinan, China, ${ }^{4}$ Shandong Provincial Qianfoshan Hospital, \\ Shandong University, Jinan, China
}

OPEN ACCESS

Edited by:

Xiaohui Fan,

Zhejiang University, China

Reviewed by:

Mark Hewitt

University of Wolverhampton,

United States

Vinícius Gonçalves Maltarollo, Federal University of Minas Gerais,

Brazil

*Correspondence:

Xiao Li

lixiao1688@163.com

Specialty section:

This article was submitted to

Predictive Toxicology,

a section of the journal

Frontiers in Pharmacology

Received: 12 October 2021 Accepted: 23 November 2021

Published: 05 January 2022

Citation:

Shi $Y$, Hua $Y$, Wang $B$, Zhang $R$ and Li $X$ (2022) In Silico Prediction and Insights Into the Structural Basis of

Drug Induced Nephrotoxicity.

Front. Pharmacol. 12:793332.

doi: 10.3389/fphar.2021.793332
Drug induced nephrotoxicity is a major clinical challenge, and it is always associated with higher costs for the pharmaceutical industry and due to detection during the late stages of drug development. It is desirable for improving the health outcomes for patients to distinguish nephrotoxic structures at an early stage of drug development. In this study, we focused on in silico prediction and insights into the structural basis of drug induced nephrotoxicity, based on reliable data on human nephrotoxicity. We collected 565 diverse chemical structures, including 287 nephrotoxic drugs on humans in the real world, and 278 non-nephrotoxic approved drugs. Several different machine learning and deep learning algorithms were employed for in silico model building. Then, a consensus model was developed based on three best individual models (RFR_QNPR, XGBOOST_QNPR, and CNF). The consensus model performed much better than individual models on internal validation and it achieved prediction accuracy of $86.24 \%$ external validation. The results of analysis of molecular properties differences between nephrotoxic and non-nephrotoxic structures indicated that several key molecular properties differ significantly, including molecular weight (MW), molecular polar surface area (MPSA), AlogP, number of hydrogen bond acceptors (nHBA), molecular solubility (LogS), the number of rotatable bonds (nRotB), and the number of aromatic rings (nAR). These molecular properties may be able to play an important part in the identification of nephrotoxic chemicals. Finally, 87 structural alerts for chemical nephrotoxicity were mined with f-score and positive rate analysis of substructures from Klekota-Roth fingerprint (KRFP). These structural alerts can well identify nephrotoxic drug structures in the data set. The in silico models and the structural alerts could be freely accessed via https://ochem.eu/article/140251 and http://www.sapredictor.cn, respectively. We hope the results should provide useful tools for early nephrotoxicity estimation in drug development.

Keywords: drug induced nephrotoxicity, in silico prediction, consensus model, structural alert, web-server

\section{INTRODUCTION}

Drug induced nephrotoxicity (DIN) can be defined as any renal injury caused directly or indirectly by medications (Sales and Foresto, 2020), which has been a major issue for patients and the pharmaceutical industry. The real world data (RWD) showed that incidence of drug induced nephrotoxicity to be approximately 14-26\% in adult populations (Mehta et al., 2004; Uchino et al., 2005; Hoste et al., 2015). 
The syndrome always contributes to considerable morbidity, mortality, and high costs, and it can lead to the development of chronic kidney disease (CKD) or end-stage renal disease (ESRD). Nephrotoxicity has become an important concern in modern drug development. It is one of the major reasons for safety-related failures at all phases of drug development and even marketed drugs being restricted or withdrawn (Zhang et al., 2019).

The mechanisms of drug induced nephrotoxicity were very complex and may be different between various drug classes. Based on the histological component of the affected kidney, the drug induced nephrotoxicity should be categorized as three mechanisms, including proximal tubular injury and acute tubular necrosis (ATN), tubular obstruction by crystals or casts containing drugs and their metabolites, and interstitial nephritis induced by drugs and their metabolites (Nolin and Himmelfarb, 2010; Kwiatkowska et al., 2021). On the other hand, the mechanisms of drug induced nephrotoxicity can also be classified according to the mode of action of the drugs, including cytotoxicity (necrosis or apoptosis), immune injury, and ischemic injury. With the help of transdermal transport system, especially organic anion transporter 1 (OAT1), drugs were accumulated in proximal convoluted tubule epithelial cells. The cell necrosis or apoptosis would be caused when high concentration was reached, then cell necrosis or apoptosis would be caused (Sekine and Endou, 2009). It was reported that drugs can act on mitochondria and block production of adenosine triphosphate (ATP), resulting in cell necrosis or apoptosis (Gai et al., 2020). Nephrotoxic drugs can also increase superoxide free radical production and decrease antioxidant free radical production in epithelial cells, which can also lead to cell necrosis or apoptosis (Paller et al., 1984). Ferroptosis was a type of cell death characterized by iron overload and accumulation of toxic lipid peroxides ( $\mathrm{Li}$ et al., 2020). In recent years, studies have shown that ferroptosis was closely related to drug induced nephrotoxicity, especially acute kidney injury, but the exact molecular biological mechanism has not been clarified, and this needs more research (Hu et al., 2019). The immune response caused by some drugs acting as antigens or haptens can result in inflammation of the blood vessels and tubules of the kidney, such as penicillin-induced interstitial nephritis (Spanou et al., 2006). This kind of immune damage was related to individual hypersensitivity to drugs, so there were significant individual differences. Besides, nephrotoxic drugs can cause renal ischemia and injury by reducing blood perfusion in renal tissues (Raza and Naureen, 2020). For example, nonsteroidal anti-inflammatory drugs (NSAIDs) were able to reduce renal blood flow by changing the resistance of glomerular entry and exit arteries, and then induce renal ischemic injury (Hörl, 2010). Unfortunately, there are few studies on the structural characteristics of nephrotoxic drugs.

The evaluation of the nephrotoxic potential of chemicals in early stage is quite important and useful for reducing the failure of drug development. The in vivo test for drug induced nephrotoxicity evaluation is always very complicated, costly, and time-consuming, and it is not suitable for screening a large number of chemicals, and especially for virtual structures. Besides, the experimental results are easily affected by various factors such as model animals and technology and environment. Compared with biological experimental methods, the use of computational toxicology for nephrotoxicity estimation of compounds has obvious advantages: (1) large quantities of compounds can be rapidly processed and predicted; (2) the toxicity of the compound can be predicted by computational models as long as the structure is known, even if the compound has not been synthesized; and (3) computational toxicological methods can also contribute to the study of the mechanisms. Consequently, it should make a lot of sense to develop fast and accurate computational tools to estimate the risk of nephrotoxicity. Over the past decades, many computational models have been developed for toxicity prediction, but only a few models reported related to kidney injury or urinary tract toxicity, and due to the variety and complexity of the symptoms and mechanisms. Lei et al. summarized the reported models related to urinary tract toxicity until 2017 (Lei et al., 2017). Most of them were established based on biomarker descriptors, and only five models were based on theoretical descriptors with the datasets of drugs in various development phases. In Lei et al.'s study, they developed a series of qualitative and quantitative structure activity relationship (QSAR) models for urinary tract toxicity prediction using 258 compounds, the best regression model reached $\mathrm{q}_{\text {ext }}^{2}$ of 0.845 for the test set, and the best classification model gave global accuracy of $90.77 \%$ for the test set. Zhang et al. developed an in silico prediction model for chemical induced urinary tract toxicity using naïve Bayes classifier based on mouse intraperitoneal data set (Zhang et al., 2019). The model provided $84.2 \%$ overall accuracy for the external test set. They also obtained several important molecular descriptors and fragments. More recently, Sun et al. developed QSAR models for screening nephrotoxicity of the ingredients in TCMs based on natural product or mixed dataset (Sun et al., 2019). The models performed well on external validation with 30 ingredients in the TCMs. The published models related to nephrotoxicity always provided high statistical performance. However, the structural characteristics of nephrotoxic and non-nephrotoxic drugs were rarely analyzed in these studies, and the usefulness of most published models was restricted because of poor availability. Besides, it should be more useful to develop the models based on real world data with human nephrotoxicity, and due to the species specificity in drug toxicity between rodent animals and human beings.

In the present study, we focused on the in silico prediction and insights into the structural basis of drug induced nephrotoxicity based on the medications causing human nephrotoxicity in the real world.

\section{MATERIALS AND METHODS}

\section{Data Source and Preparation}

In this study, only medications with human nephrotoxicity data were included. The nephrotoxic structures were extracted from the Side Effect Resource (SIDER) database (Kuhn et al., 2016). SIDER is a widely used database of adverse drug reactions (ADRs), which contained the information on approved drugs 
and the ADRs on humans. Herein, we retrieved the entire SIDER database and collected those drugs with nephrotoxicity related ADRs with frequency $\geq 0.1 \%$ in the real world. The corresponding structures of included chemicals were downloaded in smiles format from the PubChem database (Kim et al., 2016). The non-nephrotoxic structures were extracted from Zhang's work (Zhang et al., 2019). They built the negative drug dataset with the drugs without nephrotoxicity from the SIDER database. The included structures were carefully prepared as follows: (1) removing duplicate substances; (2) keep only the main ingredients in mixtures; and (3) salts were converted to their parent forms.

\section{Principal Component Analysis for the Definition of the Chemical Space}

The sufficiently structural diversity was a key issue for global QSAR models to ensure a reasonable predictive accuracy (Ancuceanu et al., 2019). Principal component analysis (PCA) is a well-known technique for reducing the dimensionality and increasing interpretability, which can solve an eigenvector problem by creating new uncorrelated variables that successively maximize variance (Jolliffe and Cadima, 2016). In this study, the chemical space of data sets was analyzed with the first two principal components of CDK (Chemistry Development Kit) Descriptors. The PCA was performed using SPSS Statistics 26.

\section{Algorithms For Model Building}

The model building was performed on the online chemical database and modeling environment (OCHEM), which is a user friendly web-based platform for automatic and simple QSAR modeling (Sushko et al., 2011). OCHEM supports the typical steps of QSAR modeling, and the models can be published and publicly used on the web (Oprisiu et al., 2013; Cui et al., 2019; Pawar et al., 2019; Cui et al., 2021; Hua et al., 2021; Huang et al., 2021; Ta et al., 2021). Among the many state-of-the-art modeling methods available on OCHEM, we applied five widely used traditional machine learning (ML) approaches and five different deep learning (DL) algorithms. As an application of artificial intelligence (AI), ML has been an effective tool for modeling in computational toxicology. Five highly effective and robust $\mathrm{ML}$ approaches were used in this study, including associative neural network (ASNN) (Tetko, 2009), support vector machine (SVM) (Chang and Lin, 2011), C4.5 decision tree (WEKA J48) (Hall et al., 2009), random forest (RFR) (Breiman, 2001), and extreme gradient boosting (XGBoost) (Chen and Guestrin, 2016). DL is an extension of machine learning, and its concept came from the research of artificial neural networks. In this study, we used five different DL approaches, including convolutional neural network fingerprint (CNF) (Tetko et al., 2019), transformer convolutional neural network (TRANSNN) (Karpov et al., 2020), TEXTCNN algorithm available from DeepChem (TEXTCNN) (Wu et al., 2017), Graph Isomorphism Network (GIN) (Capela et al., 2019), and edge attention based multirelational graph convolutional networks (EAGCNG) (Shang et al., 2018). The detailed descriptions of these ML and DL approaches can be found in the corresponding literature.

The individual parameters for each model algorithm were optimized automatically by the method itself in an inner loop of cross-validation. For instance, the SVM algorithm used libSVM (Chang and Lin, 2011) on OCHEM. There were two important configurable options for this method, including SVM type ( $\varepsilon$-SVR and $\mu$-SVR, etc.) and the kernel type (linear, polynomial, radial basis function, sigmoid, etc.). In the OCHEM workflow, classic $\varepsilon$-SVR and radial basis function kernels were used. The other SVM parameters, namely cost $\mathrm{C}$ and width of the RBF kernel $(\gamma$, g), were optimized using default grid search, and this was performed according to the LibSVM manual (Tetko et al., 2013).

\section{Molecular Description}

For the machine learning modeling, the molecular descriptors were served as the input of drug structures. We calculated eight different descriptor packages, including Chemaxon descriptors (Chemaxon, 499 descriptors), Fragmentor, GSFrag descriptors (GSFrag, 1,138 descriptors), MORDRED descriptors (MORDRED, 1826 descriptors), PyDescriptor (1,624 descriptors), QNPR descriptors (QNPR), RDKit descriptors (RDKit), and alvaDesc descriptors (5,666 descriptors). The details of these descriptor packages can be learned via http:// docs.ochem.eu//display/MAN/Molecular+descriptors.html. The descriptors were filtered with pairwise de-correlation method before the model building. There was no selection bias, since the unsupervised filtering was totally independent.

For the deep learning models, the SMILES string of each compound was served as the input without descriptors.

\section{Consensus Modeling}

Consensus modeling is to unify the prediction of unknown samples by multiple individual models to achieve a unified result, and then improve the prediction of the model. By averaging the prediction of individual models, noise can be reduced, thus, and the consensus model can provide better predictive power than most individual models alone (Tapia Garcl'a et al., 2012). Herein, the consensus model was built with simple average of predictions from the best performed individual models.

\section{Applicability Domain Assessment}

For QSAR models, it is important to estimate the applicability domain (AD) to determine whether the test compound is suitable for the specific model. In this study, we determined the AD with distance to model (DM) in OCHEM proposed by Sushko (Sushko et al., 2011). The DM assesses the distance from the target compound to the model. The larger DM means the lower applicability for the target compound.

\section{Analysis of Molecular Properties Differences Between Nephrotoxic and Non-nephrotoxic Structures}

The molecular properties of compounds always can make a significant difference in the toxicity. In this study, the analysis 
of differences of molecular properties between nephrotoxic and non-nephrotoxic structures was performed, in order to investigate the relevance of these molecular properties with drug induced nephrotoxicity. Several commonly used physicalchemical properties which have been widely adopted in the analysis for other endpoints were calculated and analyzed, including molecular weight (MW), molecular polar surface area (MPSA), AlogP, molecular solubility (LogS), the number of hydrogen bond acceptors (nHBA) and donors (nHBD), the number of rotatable bonds (nRotB), and the number of aromatic rings (nAR). These properties are relative to the molecular size, lipophilicity and solubility, and hydrogen bonding ability and complexity, respectively. The comparison between groups was tested by T-test, and the $p$ value $<0.05$ was considered to indicate statistical significance. The molecular properties were calculated with PaDEL-Descriptor package (Yap, 2011).

\section{Identification of Structural Alerts Responsible for Nephrotoxicity}

Structural alert (SA), or privileged substructure, was defined as the substructure which can cause the chemicals to become toxic. It has been well accepted in toxicity assessment, because of the direct derivation from mechanistic knowledge. SAs have been commonly used for toxicity assessment of many different endpoints (Claesson and Minidis, 2018; Yang et al., 2018; Wedlake et al., 2020). In this study, we identified the structural alerts for nephrotoxicity by calculating f-score and positive rate of each fragment from Klekota-Roth fingerprint (KRFP, 4,860 bits). The specific substructure should be regarded as a SA if presented more frequently in nephrotoxic drugs than non-nephrotoxic drugs. The positive rate (PR) of a substructure was defined as below:

$$
P R=\frac{N_{\text {fragment_positive }}}{N_{\text {fragment }}}
$$

where $\mathrm{N}_{\text {fragment_positive }}$ was the number of nephrotoxic drugs containing the fragment, and $\mathrm{N}_{\text {fragment }}$ was the total number of drugs containing the fragment.

\section{Validation and Evaluation of Models}

All the ML and DL models were first internally validated with 5-fold cross validation, and the best performed models were further validated with the external validation set. The structural alerts were assessed with the whole dataset. The classifiers and SAs were evaluated based on the counts of true positives (TP), false positives (FP), true negatives (TN), and false negatives (FN). Several statistical parameters were calculated to evaluate the classifiers, including the total accuracy (Q), sensitivity (SE), specificity (SP), enrichment factor (EF), and Matthews correlation coefficient (MCC), which were calculated with Eqs (2-6).

$$
\begin{gathered}
Q=\frac{T P+T N}{T P+F N+T N+F P} \\
S E=\frac{T P}{T P+F N}
\end{gathered}
$$

$$
\begin{gathered}
S P=\frac{T N}{T N+F P} \\
E F=\frac{T N /(T N+F N)}{(T N+F P) /(T P+F N+T N+F P)} \\
M C C=\frac{T P * T N-F P * F N}{\sqrt{(T P+F P)(T P+F N)(T N+F P)(T N+F N)}}
\end{gathered}
$$

Additionally, the receiver operating characteristic (ROC) curve was also plotted for the QSAR models, and the values of area under the ROC curve (AUC) were provided, too.

\section{RESULTS AND DISCUSSION}

\section{Data Set Analysis}

In the study, 565 diverse structures were kept after preparation, including 287 nephrotoxic drugs and 278 non-nephrotoxic drugs. As shown in Table 1, the whole data set was randomly divided into a training set with 456 chemicals (232 nephrotoxic and 224 non-nephrotoxic) and an external validation set with 109 chemicals (55 nephrotoxic and 54 non-nephrotoxic). The structures of the drugs can be found in Supplementary Table S1.

The diversity of structures is a crucial factor for the applicability of global models. Thus, we performed the principal component analysis (PCA) based on CDK descriptors to analyze the chemical space of the included compounds. PCA can simplify the complexity in high-dimensional data while retaining trends and patterns by transforming the data into fewer dimensions, which act as summaries of features (Ringnér, 2008). The first two principal components were kept and used for the definition of the chemical space of compound in the training and validation sets in this study. As shown in Figure 1, the distribution scatter diagram illustrated that the data sets shared a similar chemical space. In addition, the Tanimoto similarity index (TSI) was also calculated based on the ECFP-4 fingerprint to evaluate similarities among the structures. The average value of the entire data set was 0.13 , which indicated an evidently chemical diversity of the entire data set.

\section{Results of ML and DL Models}

Combined with five different ML algorithms and eight molecule descriptor packages, $40 \mathrm{ML}$ models were developed. Meanwhile, five DL models were developed with different algorithms using chemical SMILES-string as input. The performances of ML and DL models on 5 -fold cross validation were shown in Table 2 . The prediction accuracy $(\mathrm{Q})$ of the models ranged from 58.54 to $73.90 \%$. Among them, three models performed much better on 5 -fold cross validation than others, including a DL model (CNF) and two ML models (XGBoost_QNPR and RFR_QNPR). These models all provide good predictive ability with $\mathrm{Q}$ value $\geq 70.00 \%$ and AUC value $\geq 0.80$.

The consensus approach has been proved able to improve the accuracy of models. In this study, a consensus model was developed based on the three best models. The consensus model performed much better than the individual models. As shown in Table 2, it provided the Q value of $75.88 \%$ and the AUC 
TABLE 1 | The number of structures in the data set.

\begin{tabular}{|c|c|c|c|}
\hline & Nephrotoxic structures & Non-nephrotoxic structures & Total \\
\hline Training set & 232 & 224 & 456 \\
\hline Validation set & 55 & 54 & 109 \\
\hline Total & 287 & 278 & 565 \\
\hline
\end{tabular}

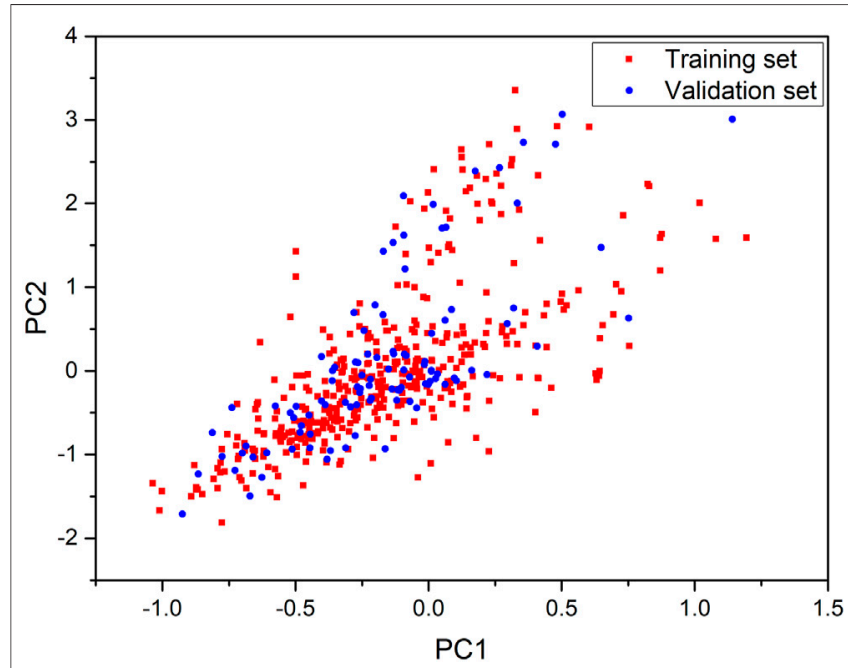

FIGURE 1 | Chemical space defined by the first two principal components of CDK descriptors. Red squares stand for the training set, blue circles stand for the validation set.

value 0.83; the values of SP, SE, EF, and MCC were 72.84, 79.01, 1.50 , and $0.52 \%$, respectively.

\section{External Validation of Models}

Due to the complete independence from the model training, the external validation set could be well used for evaluating the predictive ability of models objectively. As shown in Table 3; Figure 2, the best performed individual models also achieved good predictive results on external validation. The model developed with RFR algorithm and QNPR descriptors performed best with Q value $87.16 \%$ and AUC value 0.91 . The DL model developed with CNF algorithm also provided good predictive ability, with Q value $83.49 \%$, and AUC value 0.89 . The consensus model did not perform better than RFR_QNPR model on most of the statistical parameters, except for AUC value (0.93). It provided a $\mathrm{Q}$ value of $86.24 \%$ and MCC value of 0.82 on external validation, and the values of SE, SP, and EF were 85.45, 87.04 , and $1.72 \%$, respectively.

The results of ML model building and validation suggested that ML models developed with the QNPR descriptor performed much better than others. The QNPR descriptors were derived directly from the chemical SMILES strings. For each structure either canonical SMILES or IUPAC name would be split into fragments of a specified length determined by the configuration (Thormann et al., 2007). For the different ML algorithms, XGBoost and RFR performed better than others. XGBoost is a scalable end-to-end tree boosting system, which has been widely
TABLE 2 | Performances of models on 5-fold cross-validation.

\begin{tabular}{|c|c|c|c|c|c|c|}
\hline Model & Q (\%) & SE (\%) & SP (\%) & EF & MCC & AUC \\
\hline XGBOOST_QNPR & 72.81 & 71.98 & 73.66 & 1.46 & 0.46 & \\
\hline WEKA_J48_QNPR & 67.11 & 69.83 & & 1.37 & & \\
\hline RFR_QNPR & 72.15 & & 72.32 & 1.45 & & \\
\hline libSVMM_QNPR & 68.20 & 66.81 & 69.64 & 1.36 & & \\
\hline ASNN_QNPR & 69.30 & 68.97 & 69.64 & 1.39 & & \\
\hline XGBOO_ST_PyDescriptor & 63.82 & 62.07 & & 1.27 & & \\
\hline WEKA_J48_PyDescriptor & 60.09 & 60.78 & & 1.21 & & \\
\hline RFR_PyDescriptor & 68.8 & 69.83 & & 1.39 & & \\
\hline libSV̄M_PyDescriptor & & & & & & \\
\hline ASNN_PyDescriptor & & & & 1.27 & & \\
\hline XGBOOST_MORDRED & 64. & & & & & \\
\hline WEKA_J48_MORDRED & 67 & & & & & \\
\hline RFR_MORDRED & & & & & & \\
\hline libSV̄M_M & & & & & & \\
\hline ASNN_N & & & & & & \\
\hline GSFrag & 6 & & & & & \\
\hline WEKA_J48_GSFrag & 62. & 60. & & 24 & & \\
\hline RFR_GSFrag & 63.8 & & & 27 & & \\
\hline libSVM_GSFrag & 64. & 5 & & 26 & & \\
\hline ASNN_GSFrag & 63. & & & 24 & & \\
\hline XGBOOST_Fragmentor & & & & 26 & & \\
\hline WEKA_J48_Fragmentor & 58. & & & & & \\
\hline RFR_Fragmentor & 67 & & & 35 & & \\
\hline libSVM_Fragmentor & 67.76 & & & 35 & & \\
\hline ASNN_Fragmentor & 64.47 & & & 29 & & \\
\hline XGBOOST_ECFP4 & 63.8 & & & 1.28 & & \\
\hline WEKA_J48_ECFP4 & 59.2 & & & 1.18 & & \\
\hline RFR_ECFP4 & 66.4 & & & 1.32 & & \\
\hline libSVM_ECFP4 & 65.7 & & & 1.31 & & \\
\hline ASNN_ECFP4 & 65.5 & & & 31 & & \\
\hline XGBOOST_Chemaxon & 62.9 & & & 1.27 & & \\
\hline WEKA_J48_Chemaxon & 61. & & & 1.22 & & \\
\hline RFR_Chemaxon & & & & 1.28 & & \\
\hline libSVM_Chemaxon & 64.3 & & & 1.26 & 29 & \\
\hline ASNN_Ch & 64.9 & & & 1.31 & & \\
\hline alvaDesc & 64. & 64. & & 1.29 & & \\
\hline alvaDesc & 63. & & & 1.28 & 27 & \\
\hline RFR_alvaDesc & & & & 1.40 & & \\
\hline libSVM_alvaDesc & 65.1 & & & 33 & 30 & \\
\hline ASNN_alvaDesc & & & & 41 & & \\
\hline CNF & 73. & & & 1.45 & 48 & \\
\hline TRANSNNI & 69.4 & 65.80 & 73.2 & 1.37 & 0.39 & \\
\hline GNN GIN & 67.11 & 67.24 & 66.96 & 1.35 & 0.34 & \\
\hline EAGCNG & 58.54 & 52.42 & 64.73 & 1.15 & 0.17 & \\
\hline DEEPCHEM & 70.42 & 53.39 & 80.83 & 1.19 & 0.36 & \\
\hline Consensus & 75.88 & 72.84 & 79.02 & 1.50 & 0.52 & \\
\hline
\end{tabular}

used in many application scenarios. It supports multi-thread computation and uses regularization enhancement technology to reduce over-fitting, so as to ensure the robustness of the model. Meanwhile, it has the advantages of flexibility, fast calculation speed, and good robustness. Therefore, XGBoost is not easy to be disturbed by outliers. It can achieve state-of-the-art results on 
TABLE 3 | Performances of models on external validation.

\begin{tabular}{lcccccc}
\hline Model & Q (\%) & SE (\%) & SP (\%) & EF & MCC & AUC \\
\hline RFR_QNPR & 87.16 & 87.27 & 87.04 & 1.76 & 0.74 & 0.91 \\
XGBOOST_QNPR & 83.49 & 85.45 & 81.48 & 1.71 & 0.67 & 0.90 \\
CNF & 83.49 & 80.00 & 87.04 & 1.64 & 0.67 & 0.89 \\
Consensus & 86.24 & 85.45 & 87.04 & 1.72 & 0.72 & 0.93 \\
\hline
\end{tabular}

many machine learning challenges (Chen and Guestrin, 2016). RFR is an ensemble learning method by constructing a multitude of decision trees (DT) at training time (Breiman, 2001). It can help to improve the accuracy by reducing overfitting in decision trees and automating missing values present in the data. CNF contributed to the best DL model. CNF is one of the state-of-theart deep learning methods, which is based on ideas of text processing. It could achieve the high prediction accuracy due to the augmentation technique employed during both training and inference steps.

The DL model in our study did not show significant better predictive power compared with the ML model. However, considering that the DL models did not require additional molecular description, as long as SMILES are provided, the DL algorithms still showed obvious advantages over ML algorithms.

\section{The Differences of Molecular Properties Between Nephrotoxic and Non-nephrotoxic Drugs}

The molecular physical-chemical properties could provide useful information for biological activities. Herein, we analyzed the differences of several commonly used physical-chemical properties between nephrotoxic and non-nephrotoxic drugs. The distributions of these descriptors for nephrotoxic and non-nephrotoxic drugs can be seen in Figure 3.

The characteristics MW and MPSA can simply assess the size and complexity of compounds. For the entire data set in the study, the values of MW were distributed between 59.04 and $4,491.88$, with a mean of 416.86 . The mean value was 459.71 for nephrotoxic drugs and 372.63 for non-nephrotoxic drugs. The difference between the mean MW of nephrotoxic and nonnephrotoxic drugs was not significantly different $(p<0.01)$. The values of MPSA were distributed from 0 to 1902.88 , with a mean of 119.80 for the entire dataset. The mean value was 137.52 for nephrotoxic drugs and 101.51 for non-nephrotoxic drugs $(p<0.01)$. These results suggested that it may be significantly different in structure size and polar surface area between nephrotoxic and non-nephrotoxic compounds.

The AlogP is commonly used to represent the lipophilicity of compounds. For the entire data set, the values of AlogP ranged from -32.81 to 10.25 , with a mean of 1.48 . The mean value of AlogP was 1.34 for nephrotoxic chemicals and 1.64 for non-nephrotoxic drugs. As can be seen in Figure 3, the distributions were not significantly different between nephrotoxic and non-nephrotoxic drugs with $p$-value 0.34 . The result indicated that chemical lipophilicity may be weakly correlated with drug induced nephrotoxicity.

Chemical hydrogen bonding ability also is an important character for its activity and toxicity, and it was usually in terms of nHBA and

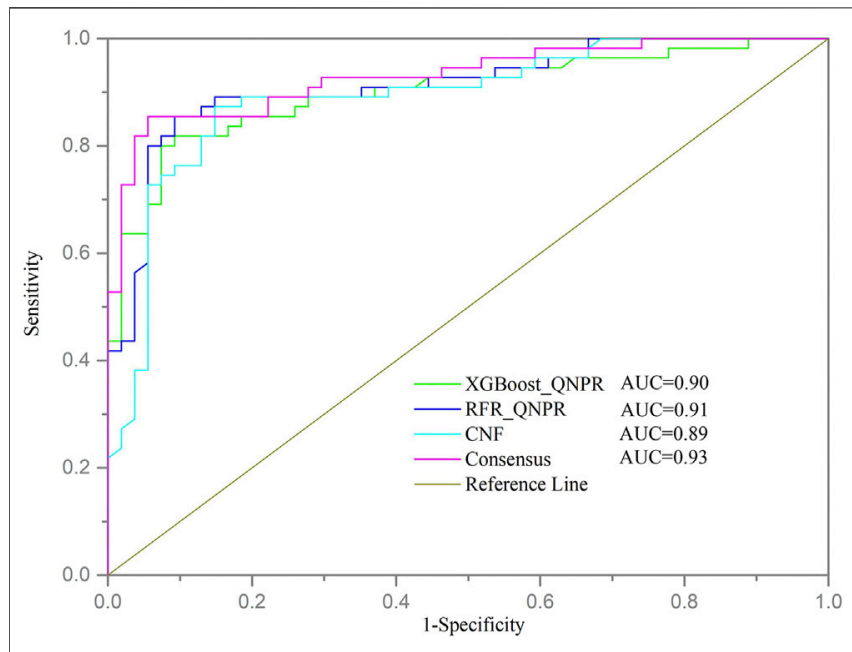

FIGURE 2 | ROC curve of models on external validation. Each color line represents a model.

nHBD. In this data set, mean values of nHBA for nephrotoxic and non-nephrotoxic drugs were 7.06 and 5.41, respectively, and the difference was significant $(p<0.01)$. Meanwhile, the nephrotoxic and non-nephrotoxic drugs had mean values of nHBD with 3.29 and 2.47 , respectively, and the difference is not significant $(p=0.07)$. The data indicated that nHBA was obviously associated with drug induced nephrotoxicity while $\mathrm{nHBD}$ was not.

$\log S$ is an estimation of molecular solubility in water. For the entire data set, the values of $\log S$ were distributed from -35.86 to 2.45 , and the mean value was -4.52 . For nephrotoxic structures, the mean value of $\log S$ was -4.81 , and it is -4.21 for non-nephrotoxic drugs. The difference also proved statistical significance $(p=0.03)$. This result demonstrated that there was difference of molecular solubility between nephrotoxic and non-nephrotoxic drugs.

As shown in Figure 3, drug induced nephrotoxicity was also obviously associated with nRotB (the mean values were 8.05 for nephrotoxic chemicals, and 5.78 for non-nephrotoxic drugs, and with $p$-value 0.01 ) and nAR (the mean values were 1.70 for nephrotoxic chemicals and 1.08 for non-nephrotoxic drugs, with significant difference $(p<0.01)$.

Through the analysis of molecular properties, several physicalchemical properties have obvious differentiating effect on drug induced nephrotoxicity. Nevertheless, nephrotoxicity is a complex endpoint yet. It's not easy to explain the mechanism of drug induced nephrotoxicity with individual simple chemical descriptors.

\section{Structural Alerts Responsible for Nephrotoxicity}

The structural alerts (SA) responsible for nephrotoxicity were identified using f-score and positive rate analysis of each fragment from KRFP fingerprint. Only the fragments existing in 6 or more drugs were kept. The fragments with $\mathrm{f}$-score $\geq 0.005$ and positive rate $\geq 0.75$ were identified. Finally, 87 representative fragments were filtered and listed in Supplementary Table S2. Among them, 16 substructures presented in nephrotoxic active chemicals only, which 

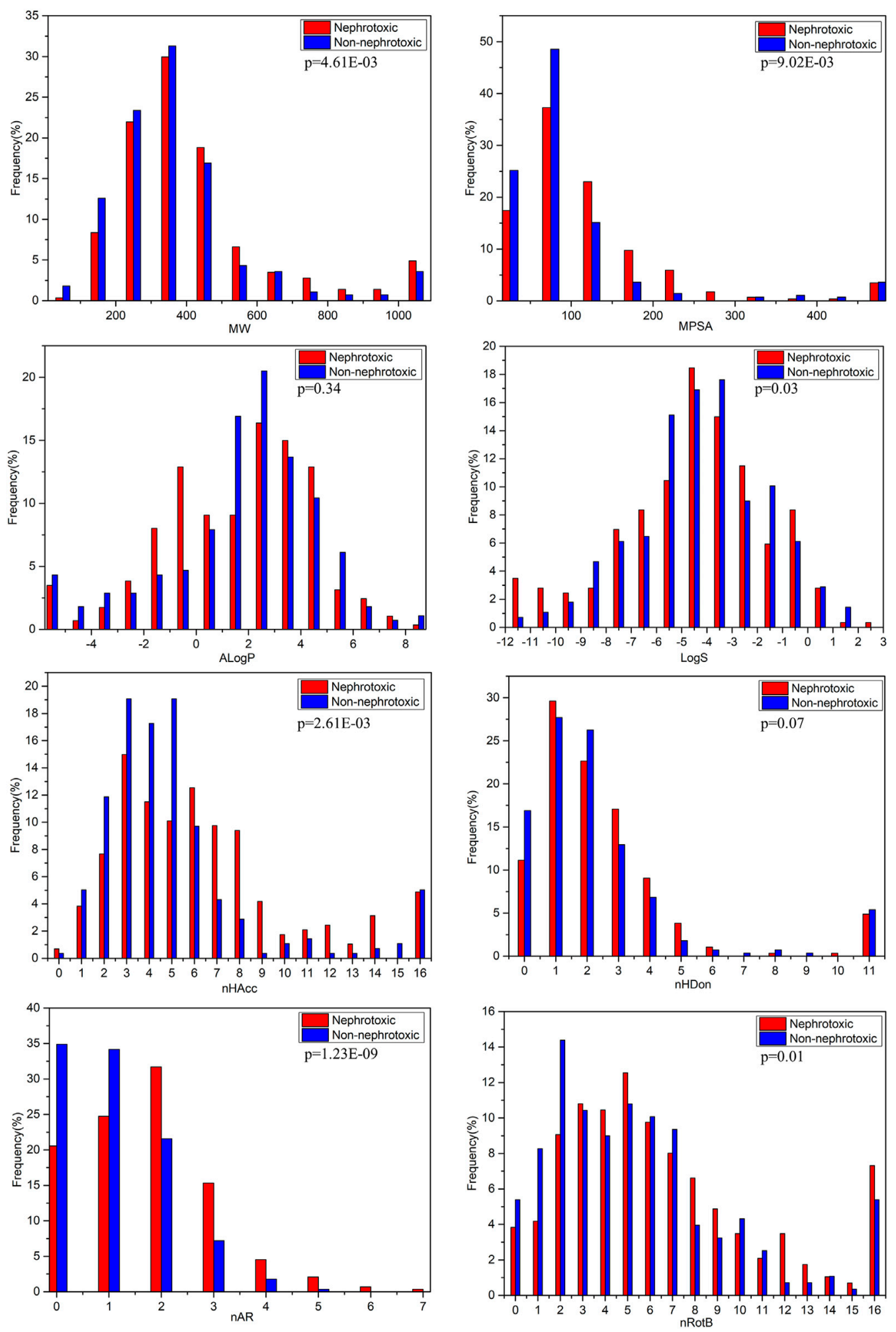

FIGURE 3 | Distributions of the commonly molecular properties for nephrotoxic and non-nephrotoxic drugs. 
TABLE 4 | Structural alerts only presented in nephrotoxic drugs.

ID

Bit

SMARTS

[!\#1][CH2][CH2]c1[CH][CH][CH][CH][CH]1

$\mathrm{KR} 413$

$[! \# 1][\mathrm{NH}] \mathrm{C}(=\mathrm{O})[\mathrm{CH}]([\mathrm{CH} 3])[\mathrm{NH}] \mathrm{C}(=\mathrm{O})[! \# 1]$

KR848

7

0

$[! \# 1] \mathrm{c} 1[\mathrm{CH}][\mathrm{CH}] \mathrm{C}(\mathrm{F})[\mathrm{CH}][\mathrm{CH}] 1$

KR1798

KR2444

[!\#1]N1[CH2][CH2]N([CH3])[CH2][CH2]1

0

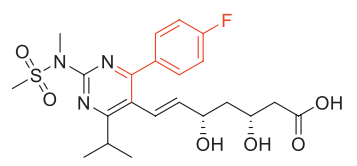

4

(

5

KR3206

c1nc2ccccc2[nH]1

6

KR3280

$\mathrm{CC}(=\mathrm{O}) \operatorname{c1} \operatorname{ccc}(\mathrm{N}) \mathrm{cc} 1$

7

KR3540

$\operatorname{Cc1} \operatorname{ccc}(\operatorname{cc} 1) \operatorname{cocccc} 2$

Cc1ccc(F)cc1

8

KR3548

Coce(F)ect

8

9

KR3586
$\mathrm{Cc} 1 \mathrm{cccc}(\mathrm{F}) \mathrm{c} 1$
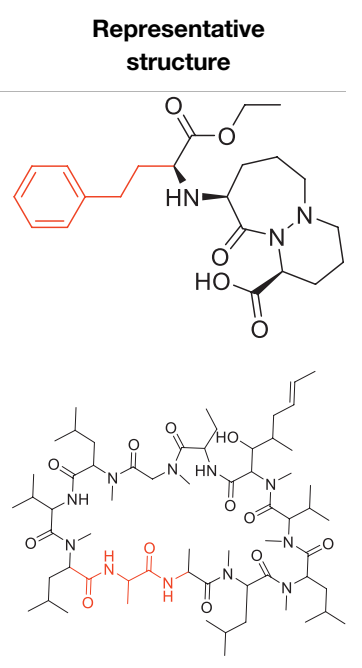

6

0

0<smiles>CC1COc2c(N3CCN(C)CC3)c(F)cc3c(=O)c(C(=O)O)cn1c23</smiles>

7

0

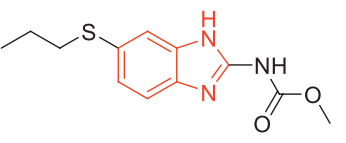<smiles>COc1c(N2CCNC(C)C2)c(F)cc2c(=O)c(C(=O)O)cn(C3CC3)c12</smiles>

7

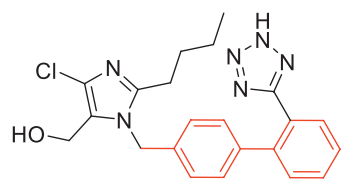

0

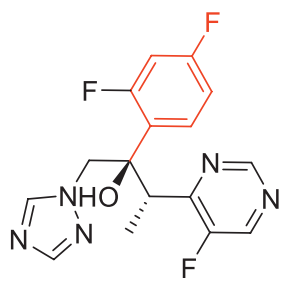

8

0

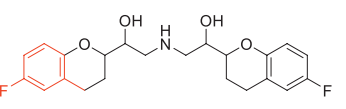

(Continued on following page) 
TABLE 4 | (Continued) Structural alerts only presented in nephrotoxic drugs.

\begin{tabular}{|c|c|c|c|c|c|}
\hline ID & Bit & SMARTS & Positive & Negative & $\begin{array}{l}\text { Representative } \\
\text { structure }\end{array}$ \\
\hline 10 & KR4029 & $\mathrm{CS}(\mathrm{c} 1 \mathrm{nc} 2 \mathrm{ccccc} 2[\mathrm{nH}] 1)$ & 6 & 0 & \\
\hline 11 & KR4064 & $\mathrm{Fc} 1 \mathrm{cccc}(\mathrm{C}=\mathrm{O}) \mathrm{c} 1$ & 8 & 0 & \\
\hline 12 & KR4065 & $\mathrm{Fc} 1 \mathrm{cccc}(\mathrm{F}) \mathrm{c} 1$ & 8 & 0 & \\
\hline 14 & KR4252 & $\operatorname{Nc1} 1 \mathrm{ccc}(\mathrm{F}) \mathrm{cc} 1$ & 11 & 0 & \\
\hline 15 & KR4556 & $\mathrm{O}=\mathrm{CNCCCCNC}=\mathrm{O}$ & 8 & 0 & \\
\hline
\end{tabular}

covered 76 nephrotoxic drugs. Details of each fragment and the representative structures were shown in Table 4.

We analyzed the structural characteristics of drugs in the entire data set, and $75.04 \%$ structures (424/565) were correctly classified. Among the 290 drug structures contained at least one identified substructure, 218 structures were true nephrotoxic. The classification accuracy was $75.17 \%$. For the drug structures that did not contain any identified substructure, 206 of 275 drugs (74.91\%) were true non-nephrotoxic. The frequencies of these substructures were much higher in nephrotoxic drugs than non-nephrotoxic drugs, and showed a good ability to distinguish nephrotoxic drugs in the whole data set. To a certain degree, these fragments could be considered as the structural alerts responsible for nephrotoxicity. If a structure contains one or more structural alerts, it is more likely to be nephrotoxic than non-nephrotoxic.

Fluorine is widely used in medicinal chemistry to improve a molecule's potency and permeability. However, the metabolism of fluorinated compounds may produce fluoride and other toxic metabolites (Pan, 2019). As shown in Table 4, several selected structural alerts contained phenyl fluoride (Nos 3, 8, 9, 11, 12, and 14). Since the kidney is a main target organ of mammalian fluoride systemic exposure and renal toxicity can occur after acute and chronic fluoride intoxication (Quadri et al., 2016). The kidney plays a vital role in fluoride metabolism, since $50-80 \%$ of fluoride is removed via urinary excretion. Fluorinated compounds can be toxic to the kidneys in a number of ways. It has widely been reported that fluorinated compounds can increase the generation of reactive oxygen species (ROS) and free radicals, cause extensive oxidative stress and excessive lipid peroxidation, and reduce antioxidant enzyme activities in vivo or in vitro (Quadri et al., 2016). Thus, numerous renal structural, ultrastructural, and functional may be changed after receiving increased amounts of fluorinated compounds exposure. Certainly, this is not meant to sound the alarm on all fluorinated compounds, we suggest raising the awareness of common drug instability and metabolism issues leading to defluorination, as well as the resulting reactive/toxic metabolite (Pan, 2019). Polyamines and their derivatives (Nos 2 and 15) 
widely existed in aminoglycosides and the other nephrotoxic drugs. Polyamines catabolism can be stimulated through oxidation, which will lead to the generation of ROS and low abundance of free polyamines with antioxidant capacity (Pegg, 2013; Murray Stewart et al., 2018). Consequently, this process is associated with a number of pathologies, including cancer, neurological disorders, and kidney dysfunction. Recent studies have also suggested a role for polyamines derivatives in the p53mediated ferroptotic response to ROS stress. It is an irondependent and nonapoptotic mode of cell death and has been associated with drug-induced nephrotoxicity recently (Ou et al., 2016; Murray Stewart et al., 2018). Benzimidazoles derivatives were always proposed as new bioreductive prodrugs with the potential anticancer activity, due to their effect on the DNA destruction and growth inhibition into selected tumor cell lines (Błaszczak-Świątkiewicz et al., 2014). Besides, benzimidazoles derivatives also can induce concurrent apoptotic and pyroptotic cell death (Ren et al., 2021). However, when these processes take place in kidney cells, kidney damage can occur. Toluene-contained structures (Nos 1 and 7) were also selected as structural alerts for drug induced rhabdomyolysis (Cui et al., 2019), which always associated to oliguric renal failure. In the setting of toluene intoxication, electrolyte disturbances may play important roles on causing rhabdomyolysis (Camara-Lemarroy et al., 2015). Toluene has also been associated with direct induction of acute tubular necrosis and acute oliguric renal failure. Among the SAs we identified, there were several fragments that also could cause the formation of ROS (Nos 6 and 16 , etc.), which may contribute to the nephrotoxicity.

In the present study, the SAs were identified with the f-score and frequency analysis of defined substructures. These methods have been widely used for the SA discovery for many other endpoints. In fact, these methods have some shortcomings. They are not able to characterize the spatial arrangement of identified substructures, and they cannot make a good distinction when more than one SA presented in the same structure. In spite of this, these structural alerts were able to well distinguish chemical structures with renal toxicity, and they can help to understand the specific fragments which lead to nephrotoxicity. Therefore, these SAs should be severed as a useful tool to visually evaluate the nephrotoxicity of chemicals.

\section{Availability of QSAR Models and Structural Alerts}

For ease of use, the QSAR models were made available at OCHEM. The consensus model could be accessed via https:// ochem.eu/article/140251. The three best individual models (RFR_QNPR, XGBOOST_QNPR, and CNF) were also available with the corresponding model IDs. Users can predict chemical nephrotoxicity by using the "Apply the model to new compounds" link. In addition, the data sets for modeling could be downloaded by using the "Export this basket" link.

The structural alerts responsible for nephrotoxicity have been integrated as part of our Web server SApredictor, which is a structural alert based expert system for drug toxicity prediction and freely available at http://www.sapredictor.cn. With the help of SApredictor, people can quickly evaluate whether the query chemicals are nephrotoxic, and the specific structural fragments that lead to the nephrotoxicity of the compounds will be intuitively shown to provide valuable reference for the modification of the structures.

\section{CONCLUSION}

In this study, we collected 287 drug structures which were proved nephrotoxic on humans in the real world. A comparable amount of non-nephrotoxic structures was also extracted from approved drugs. Then, in silcio models were developed using OCHEM tools. A total of $40 \mathrm{ML}$ models were developed using 5 different machine learning algorithms along with 8 descriptor packages. Besides, 5 DL models were also developed using different deep learning methods. Among them, two ML models (RFR_QNPR and XGBoost_QNPR), and one DL models (CNF) provided best predictive ability. A consensus model was developed based on them, which performed much better on internal validation, and provided good predictive ability on external validation. The consensus model and the best individual models were freely available at https://ochem.eu/article/140251. Moreover, the differences of several commonly used physical-chemical properties between nephrotoxic and non-nephrotoxic drugs were investigated. The results indicated that several key molecular properties differ significantly between nephrotoxic and nonnephrotoxic structures, including molecular weight (MW), molecular polar surface area (MPSA), AlogP, number of hydrogen bond acceptors (nHBA), molecular solubility (LogS), the number of rotatable bonds (nRotB), and the number of aromatic rings (nAR). Thus, these molecular descriptors may be associated to drug-induced nephrotoxicity and could play an important role in the identification of nephrotoxic chemicals. Finally, we identified the structural alerts responsible for nephrotoxicity using f-score and positive rate analysis. There were 87 structural alerts identified from the fragments of KRFP fingerprint. A compound would be classified as nephrotoxic if it contains one or more such SAs. These structural alerts showed a good ability to distinguish nephrotoxic drugs in the entire data set. They have been integrated as part of our web server SApredictor, which is freely available at www.sapredictor.cn. The in silico models and the structural alerts could be useful tools for estimation of nephrotoxicity in drug discovery.

\section{DATA AVAILABILITY STATEMENT}

The original contributions presented in the study are included in the article/Supplementary Materials, and further inquiries can be directed to the corresponding author.

\section{AUTHOR CONTRIBUTIONS}

XL contributed to conception and design of the study. YS and YH collected the datasets and carried out the experiments. YS, $\mathrm{BW}$, and RZ performed the analysis. YS, $\mathrm{YH}$, and $\mathrm{XL}$ 
interpreted the results and wrote the manuscript. All authors contributed to manuscript revision, read, and approved the submitted version.

\section{FUNDING}

This work was supported by the National Natural Science Foundation of China (Grant 81803433) and the Special Research project of Clinical Toxicology of Chinese Society of Toxicology (CST2020CT104).

\section{REFERENCES}

Ancuceanu, R., Dinu, M., Neaga, I., Laszlo, F. G., and Boda, D. (2019). Development of QSAR Machine Learning-Based Models to Forecast the Effect of Substances on Malignant Melanoma Cells. Oncol. Lett. 17 (5), 4188-4196. doi:10.3892/ol.2019.10068

Błaszczak-Świątkiewicz, K., Olszewska, P., and Mikiciuk-Olasik, E. (2014). Biological Approach of Anticancer Activity of New Benzimidazole Derivatives. Pharmacol. Rep. 66 (1), 100-106. doi:10.1016/j.pharep.2014.01.001

Breiman, L. (2001). Random Forests. Machine Learn. 45 (1), 5-32. doi:10.1023/a: 1010933404324

Camara-Lemarroy, C. R., Rodríguez-Gutiérrez, R., Monreal-Robles, R., and González-González, J. G. (2015). Acute Toluene Intoxication-Clinical Presentation, Management and Prognosis: a Prospective Observational Study. BMC Emerg. Med. 15 (1), 19. doi:10.1186/s12873-015-0039-0

Capela, F., Nouchi, V., Van Deursen, R., Tetko, I. V., and Godin, G. (2019). Multitask Learning on Graph Neural Networks Applied to Molecular Property Predictions. Available at: https://ui.adsabs.harvard.edu/abs/ 2019arXiv191013124C (Accessed October 01, 2019).

Chang, C.-C., and Lin, C.-J. (2011). LIBSVM: A Library for Support Vector Machines. ACM Trans. Intell. Syst. Technol. 2, 1-27. doi:10.1145/ 1961189.1961199

Chen, T., and Guestrin, C. (2016). "XGBoost: A Scalable Tree Boosting System", in: Proceedings of the 22nd ACM SIGKDD International Conference on Knowledge Discovery and Data Mining. San Francisco, California, USA: Association for Computing Machinery.

Claesson, A., and Minidis, A. (2018). Systematic Approach to Organizing Structural Alerts for Reactive Metabolite Formation From Potential Drugs. Chem. Res. Toxicol. 31 (6), 389-411. doi:10.1021/acs.chemrestox.8b00046

Cui, X., Liu, J., Zhang, J., Wu, Q., and Li, X. (2019). In Silico prediction of DrugInduced Rhabdomyolysis With Machine-Learning Models and Structural Alerts. J. Appl. Toxicol. 39 (8), 1224-1232. doi:10.1002/jat.3808

Cui, X., Yang, R., Li, S., Liu, J., Wu, Q., and Li, X. (2021). Modeling and Insights Into Molecular Basis of Low Molecular Weight Respiratory Sensitizers. Mol. Divers. 25 (2), 847-859. doi:10.1007/s11030-020-10069-3

Gai, Z., Gui, T., Kullak-Ublick, G. A., Li, Y., and Visentin, M. (2020). The Role of Mitochondria in Drug-Induced Kidney Injury. Front. Physiol. 11, 1079. doi:10.3389/fphys.2020.01079

Hall, M., Frank, E., Holmes, G., Pfahringer, B., Reutemann, P., and Witten, I. H. (2009). The WEKA Data Mining Software. SIGKDD Explor. Newsl. 11 (1), 10-18. doi:10.1145/1656274.1656278

Hörl, W. H. (2010). Nonsteroidal Anti-Inflammatory Drugs and the Kidney. Pharmaceuticals. 3, 2291. doi:10.3390/ph3072291

Hoste, E. A., Bagshaw, S. M., Bellomo, R., Cely, C. M., Colman, R., Cruz, D. N., et al. (2015). Epidemiology of Acute Kidney Injury in Critically Ill Patients: the Multinational AKI-EPI Study. Intensive Care Med. 41 (8), 1411-1423. doi:10.1007/s00134-015-3934-7

Hu, Z., Zhang, H., Yang, S. K., Wu, X., He, D., Cao, K., et al. (2019). Emerging Role of Ferroptosis in Acute Kidney Injury. Oxid Med. Cell Longev. 2019, 8010614. doi:10.1155/2019/8010614

\section{ACKNOWLEDGMENTS}

The authors gratefully acknowledge the encouragement and support from Miss Chaoyue Yang.

\section{SUPPLEMENTARY MATERIAL}

The Supplementary Material for this article can be found online at: https://www.frontiersin.org/articles/10.3389/fphar.2021.793332/ full\#supplementary-material

Hua, Y., Shi, Y., Cui, X., and Li, X. (2021). In Silico prediction of Chemical-Induced Hematotoxicity with Machine Learning and Deep Learning Methods. Mol. Divers. 25 (3), 1585-1596. doi:10.1007/s11030-021-10255-X

Huang, X., Tang, F., Hua, Y., and Li, X. (2021). In Silico Prediction of DrugInduced Ototoxicity Using Machine Learning and Deep Learning Methods. Chem. Biol. Drug Des. 98 (2), 248-257. doi:10.1111/cbdd.13894

Jolliffe, I. T., and Cadima, J. (2016). Principal Component Analysis: a Review and Recent Developments. Philos. Trans. A. Math. Phys. Eng. Sci. 374 (2065), 20150202. doi:10.1098/rsta.2015.0202

Karpov, P., Godin, G., and Tetko, I. V. (2020). Transformer-CNN: Swiss Knife for QSAR Modeling and Interpretation. J. Cheminform. 12 (1), 17. doi:10.1186/ s13321-020-00423-w

Kim, S., Thiessen, P. A., Bolton, E. E., Chen, J., Fu, G., Gindulyte, A., et al. (2016). PubChem Substance and Compound Databases. Nucleic Acids Res. 44 (D1), D1202-D1213. doi:10.1093/nar/gkv951

Kuhn, M., Letunic, I., Jensen, L. J., and Bork, P. (2016). The SIDER Database of Drugs and Side Effects. Nucleic Acids Res. 44 (D1), D1075-D1079. doi:10.1093/ nar/gkv1075

Kwiatkowska, E., Domański, L., Dziedziejko, V., Kajdy, A., Stefańska, K., Kwiatkowski, S., et al. (2021). The Mechanism of Drug Nephrotoxicity and the Methods for Preventing Kidney Damage. Int. J. Mol. Sci. 22 (11), 6109. doi:10.3390/ijms22116109

Lei, T., Sun, H., Kang, Y., Zhu, F., Liu, H., Zhou, W., et al. (2017). ADMET Evaluation in Drug Discovery. 18. Reliable Prediction of Chemical-Induced Urinary Tract Toxicity by Boosting Machine Learning Approaches. Mol. Pharm. 14 (11), 3935-3953. doi:10.1021/acs.molpharmaceut.7b00631

Li, J., Cao, F., Yin, H. L., Huang, Z. J., Lin, Z. T., Mao, N., et al. (2020). Ferroptosis: Past, Present and Future. Cell Death Dis. 11 (2), 88. doi:10.1038/s41419-0202298-2

Mehta, R. L., Pascual, M. T., Soroko, S., Savage, B. R., Himmelfarb, J., Ikizler, T. A., et al. (2004). Spectrum of Acute Renal Failure in the Intensive Care Unit: The PICARD Experience. Kidney Int. 66 (4), 1613-1621. doi:10.1111/j.15231755.2004.00927.x

Murray Stewart, T., Dunston, T. T., Woster, P. M., and Casero, R. A., Jr (2018). Polyamine Catabolism and Oxidative Damage. J. Biol. Chem. 293 (48), 18736-18745. doi:10.1074/jbc.TM118.003337

Nolin, T. D., and Himmelfarb, J. (2010). Mechanisms of Drug-Induced Nephrotoxicity. Adverse Drug React. 196, 111-130. doi:10.1007/978-3-642 00663-0_5

Oprisiu, I., Novotarskyi, S., and Tetko, I. V. (2013). Modeling of NonAdditive Mixture Properties Using the Online CHEmical Database and Modeling Environment (OCHEM). J. Cheminform. 5 (1), 4. doi:10.1186/ 1758-2946-5-4

Ou, Y., Wang, S. J., Li, D., Chu, B., and Gu, W. (2016). Activation of SAT1 Engages Polyamine Metabolism With P53-Mediated Ferroptotic Responses. Proc. Natl. Acad. Sci. U S A. 113 (44), E6806. doi:10.1073/pnas.1607152113

Paller, M. S., Hoidal, J. R., and Ferris, T. F. (1984). Oxygen Free Radicals in Ischemic Acute Renal Failure in the Rat. J. Clin. Invest. 74 (4), 1156-1164. doi:10.1172/JCI111524

Pan, Y. (2019). The Dark Side of Fluorine. ACS Med. Chem. Lett. 10 (7), 1016-1019. doi:10.1021/acsmedchemlett.9b00235 
Pawar, G., Madden, J. C., Ebbrell, D., Firman, J. W., and Cronin, M. T. D. (2019). Silico Toxicology Data Resources to Support Read-Across and (Q)SAR. Front. Pharmacol. 10, 561. doi:10.3389/fphar.2019.00561

Pegg, A. E. (2013). Toxicity of Polyamines and Their Metabolic Products. Chem. Res. Toxicol. 26 (12), 1782-1800. doi:10.1021/tx400316s

Quadri, J. A., Alam, M. M., Sarwar, S., Singh, S., Shariff, A., and Das, T. K. (2016). Fluoride Induced Nephrotoxicity: Apoptosis, Ultrastructural Changes and Renal Tubular Injury in Experimental Animals. Int. J. Ayurveda Pharma Res. 4, 91-95.

Raza, Z., and Naureen, Z. (2020). Melatonin Ameliorates the Drug Induced Nephrotoxicity: Molecular Insights. Nefrologia. 40 (1), 12-25. doi:10.1016/ j.nefro.2019.06.009

Ren, L.-w., Li, W., Zheng, X.-j., Liu, J.-y., Yang, Y.-h., Li, S., et al. (2021). Benzimidazoles Induce Concurrent Apoptosis and Pyroptosis of Human Glioblastoma Cells via Arresting Cell Cycle. Acta Pharmacologica Sinica. doi:10.1038/s41401-021-00752-y

Ringnér, M. (2008). What Is Principal Component Analysis? Nat. Biotechnol. 26 (3), 303-304. doi:10.1038/nbt0308-303

Sales, G. T. M., and Foresto, R. D. (2020). Drug-Induced Nephrotoxicity. Rev. Assoc. Med. Bras. 66 (Suppl. 1), s82-s90. doi:10.1590/1806-9282.66.s1.82

Sekine, T., and Endou, H. (2009). Children's Toxicology from Bench to Bed-Druglinduced Renal Injury (3): Drug Transporters and Toxic Nephropathy in Childhood. J. Toxicol. Sci. 34, SP259-65. doi:10.2131/jts.34.SP259

Shang, C., Liu, Q., Chen, K.-S., Sun, J., Lu, J., Yi, J., et al. (2018). Edge Attention-Based Multi-Relational Graph Convolutional Networks. Available. Available at: https://ui. adsabs.harvard.edu/abs/2018arXiv180204944S (Accessed February 01, 2018).

Spanou, Z., Keller, M., Britschgi, M., Yawalkar, N., Fehr, T., Neuweiler, J., et al. (2006). Involvement of Drug-Specific T Cells in Acute Drug-Induced Interstitial Nephritis. J. Am. Soc. Nephrol. 17 (10), 2919-2927. doi:10.1681/ ASN.2006050418

Sun, Y., Shi, S., Li, Y., and Wang, Q. (2019). Development of Quantitative Structure-Activity Relationship Models to Predict Potential Nephrotoxic Ingredients in Traditional Chinese Medicines. Food Chem. Toxicol. 128, 163-170. doi:10.1016/j.fct.2019.03.056

Sushko, I., Novotarskyi, S., Körner, R., Pandey, A. K., Rupp, M., Teetz, W., et al. (2011). Online Chemical Modeling Environment (OCHEM): Web Platform for Data Storage, Model Development and Publishing of Chemical Information. J. Comput. Aided Mol. Des. 25 (6), 533-554. doi:10.1007/s10822-011-9440-2

Ta, G. H., Weng, C.-F., and Leong, M. K. (2021). In Silico Prediction of Skin Sensitization: Quo Vadis? Front. Pharmacol. 12, 655771. doi:10.3389/ fphar.2021.655771

Tapia Garci'a, J. M., del Moral, M. J., Martínez, M. A., and Herrera-Viedma, E. (2012). A Consensus Model for Group Decision Making Problems with Linguistic Interval Fuzzy Preference Relations. Expert Syst. Appl. 39 (11), 10022-10030. doi:10.1016/j.eswa.2012.02.008

Tetko, I. V. (2009). "Associative Neural Network," in Artificial Neural Networks: Methods and Applications. Editors D. J. Livingstone, and (Totowa, NJ: Humana Press), 180-197.
Tetko, I. V., Novotarskyi, S., Sushko, I., Ivanov, V., Petrenko, A. E., Dieden, R., et al. (2013). Development of Dimethyl Sulfoxide Solubility Models Using 163,000 Molecules: Using a Domain Applicability Metric to Select More Reliable Predictions. J. Chem. Inf. Model. 53 (8), 1990-2000. doi:10.1021/ci400213d

Tetko, I. V., Karpov, P., Bruno, E., Kimber, T. B., and Godin, G. (2019). Augmentation Is What You Need!. Lecture Notes Computer Sci. 11731, 831. doi:10.1007/978-3-030-30493-5_79

Thormann, M., Vidal, D., Almstetter, M., and Pons, M. (2007). Nomen Est Omen: Quantitative Prediction of Molecular Properties Directly from IUPAC Names. Toainfoj. 1, 28-32. doi:10.2174/1874136300701010028

Uchino, S., Kellum, J. A., Bellomo, R., Doig, G. S., Morimatsu, H., Morgera, S., et al. (2005). Acute Renal Failure in Critically Ill Patients: a Multinational, Multicenter Study. JAMA. 294 (7), 813-818. doi:10.1001/jama.294.7.813

Wedlake, A. J., Allen, T. E. H., Goodman, J. M., Gutsell, S., Kukic, P., and Russell, P. J. (2020). Confidence in Inactive and Active Predictions From Structural Alerts. Chem. Res. Toxicol. 33 (12), 3010-3022. doi:10.1021/acs.chemrestox.0c00332

Wu, Z., Ramsundar, B., Feinberg, E. N., Gomes, J., Geniesse, C., Pappu, A. S., et al. (2017). MoleculeNet: A Benchmark for Molecular Machine Learning. Available. Available at: https:/ui.adsabs.harvard.edu/abs/ 2017arXiv170300564W (Accessed March 01, 2017).

Yang, H., Sun, L., Li, W., Liu, G., and Tang, Y. (2018). Identification of Nontoxic Substructures: A New Strategy to Avoid Potential Toxicity Risk. Toxicol. Sci. 165 (2), 396-407. doi:10.1093/toxsci/kfy 146

Yap, C. W. (2011). PaDEL-descriptor: An Open Source Software to Calculate Molecular Descriptors and Fingerprints. J. Comput. Chem. 32 (7), 1466-1474. doi:10.1002/jcc. 21707

Zhang, H., Ren, J. X., Ma, J. X., and Ding, L. (2019). Development of an In Silico Prediction Model for Chemical-Induced Urinary Tract Toxicity by Using Naïve Bayes Classifier. Mol. Divers. 23 (2), 381-392. doi:10.1007/s11030-018-9882-8

Conflict of Interest: The authors declare that the research was conducted in the absence of any commercial or financial relationships that could be construed as a potential conflict of interest.

Publisher's Note: All claims expressed in this article are solely those of the authors and do not necessarily represent those of their affiliated organizations, or those of the publisher, the editors, and the reviewers. Any product that may be evaluated in this article, or claim that may be made by its manufacturer, is not guaranteed or endorsed by the publisher.

Copyright ( 2022 Shi, Hua, Wang, Zhang and Li. This is an open-access article distributed under the terms of the Creative Commons Attribution License (CC BY). The use, distribution or reproduction in other forums is permitted, provided the original author(s) and the copyright owner(s) are credited and that the original publication in this journal is cited, in accordance with accepted academic practice. No use, distribution or reproduction is permitted which does not comply with these terms. 